\title{
A comparison of quality of life of obstetric fistula survivors in Zimbabwe before and after surgical treatment
}

\author{
Chipo Chimamise ${ }^{1^{*}} \mathbb{D}$, Iris Shiripinda' ${ }^{1}$ Stephen P. Munjanja ${ }^{2}$ and Mazvita Machinga ${ }^{1}$
}

\begin{abstract}
Background: In Zimbabwe, repair of obstetric fistula was established as a public health intervention in 2015. The aim of this study was to assess the quality life of obstetric fistula survivors before and after surgical repair of the fistula.

Methods: A longitudinal, before and after cohort study was conducted using the WHOQOL-BREF tool to assess quality of life before and after surgical treatment of obstetric fistula. The tool assess general health, experience of life in general, physical health, psychological health, social and environmental health. Data were analyzed using SPSS version 16.0 for descriptive measures and significance.

Results: Of the 29 women who came for obstetric fistula repair at the center in November and December 2019, 26 were enrolled into the study. All participants had transvaginal fistula repair and 24 had successful repair i.e. the fistulas were closed. Two of them still had stress incontinence by the time of data collection. The post treatment mean scores, using the WHOQOL assessment tool, on physical, psychological, social, environmental and general health significantly improved from the pretreatment mean scores. There was no significant change in some facets of the quality of life domains such as financial resources, opportunities for participation in leisure activities and dependence on medicines.

Conclusions: This study concluded that surgical treatment of obstetric fistula improves the quality of life of survivors significantly and recommends that untreated fistula survivors be identified and linked to care and treatment.
\end{abstract}

Keywords: Quality of life, Obstetric fistula survivors, Urinary incontinence, Zimbabwe

\section{Background}

Obstetric fistula has been described as the most devastating birth injury by the World Health Organization [1] and the United Nations Population Fund [2]. It is an abnormal communication between the urinary tract or the gastrointestinal tract and the genital tract, produced by obstetric causes, usually prolonged and obstructed labor [3]. There are different types of obstetric fistula but the most common type is vesico-vaginal fistula [4]. Vesico-vaginal fistula (VVF), is an abnormal connection

\footnotetext{
*Correspondence: chipochimamise@gmail.com

${ }^{1}$ Department of Health Sciences, College of Health, Agriculture

and Natural Sciences, Africa University, Mutare, Zimbabwe

Full list of author information is available at the end of the article
}

between the vagina and the bladder leading to urinary incontinence.

According to the International Continence Society (ICS), urinary incontinence is the involuntary and uncontrollable leakage of urine causing physical discomfort and problems due to maintenance of feminine hygiene [4]. This leads to affected women altering their ways of life in the process of coping with this problem thereby their quality of life is greatly affected [5]. Although urinary incontinence is not life threatening by itself, it brings anxiety, distress, loss of self-esteem and affects woman's social, cultural, economic, domestic, physical, marital and sexual well-being [6].

Quality of life refers to the degree to which a person enjoys the possibilities of his or her life and includes both objective and subjective indicators. It is a reflection of 
individual's sense of well-being and satisfaction with life [7].

The World Health Organization (WHO) described quality of life as individuals' perceptions of their position in life in the context of the culture and value systems in which they live and in relation to their goals, expectations, standards and concerns [8].

As such, quality of life cannot be taken as equal to health status, life style or wellbeing $[1,5]$. Measurement of quality of life had been lacking in research and was described as the missing measurement in health [9].

Studies documenting signs and symptoms and objective urodynamic data provide needed information for treatment and management of fistula patients, but the evidence of the impact of obstetric fistula on affected women's lives may be inadequate [4]. Comprehensive care (including identification of affected women, surgical treatment, rehabilitation and reintegration back into society), of fistula survivors is crucial if improvement of quality of care is to be achieved through fistula care and management programs. Conducting quality of life assessments in health care redirects attention and interventions to address the human aspect of patients' well-being [8].

In Zimbabwe, the obstetric fistula repair program was started at a public health level in 2015. From August 2015, fistula repairs have been conducted through quarterly camps and targets 60 women per camp. Because the program predominantly makes use of visiting surgeons, patients are operated on in groups of 60 every 3 months. These are termed fistula repair camps. More than 700 women have been repaired for obstetric fistula since the start of the program up to end of 2019. Of concern is that the quality of life of these women has not been assessed and documented and the question on whether there was any change in the quality of life of obstetric fistula survivors after treatment remained crucial.

The aim of this study was to assess and compare quality of life of women affected by obstetric fistula before and after surgical treatment for the cohort of women who attended the October to December 2019 fistula repair camp in Zimbabwe.

\section{Methods}

\subsection{Study design}

A longitudinal before and after study using the standard WHOQOL-BREF tool for assessing quality of life of study participants before and after fistula repair was conducted between November 2019 and August 2020.

\subsection{Study setting and study participants}

The data collection for quality of life before surgical repair of study participants was conducted at the national center for obstetric fistula repair in Zimbabwe. Women who had come for obstetric fistula repair at the hospital were the participants of this study. The diagnosis of fistula was first suspected at health facilities in the women's communities by nurses based on signs and symptoms using the community fistula screening tool (Additional file 1). The women were referred to the fistula center where physical per vagina and speculum examinations including dye tests were conducted to verify the presence, site and extend of the fistula. The second phase of data collection was conducted 6 months after repair, per telephone for the majority of the participants and through home visits for three participants.

\subsection{Inclusion criteria}

Women who had obstetric fistula of any type and any duration and who had come for fistula care at the fistula repair center were the participants of the. Those women who were willing to participate and gave written informed consent for both the baseline and the follow-up interviews, were eligible for the study.

\subsection{Exclusion criteria}

Obstetric fistula survivors who were not willing to participate in the study.

\subsection{Data collection}

Data collection for the "before" part of the study was conducted between 18 November and 6 December 2019. To allow adequate time for preparations for surgery, obstetric fistula patients are admitted at the center about 3 days prior to the actual day of operation. It was during this waiting time that participants were recruited into the study and data for assessing quality of life before repair was collected. The purpose of the study and procedures were explained to all the twenty-nine women who had come for fistula repair. Written information about the study was also distributed to all the women for them to read and understand. On the day of actual data collection for each participant, all aspects of the study were again explained to each individual woman, including procedures, benefits, advantages, disadvantages and need for follow-up data collection. The need for the follow-up interview, which would be conducted at least 4 months after fistula repair when they come for their review consultations, through home visits or per telephone was also explained.

Informed written consent for participation in the study was obtained from all participants. Data was collected using the WHOQOL_BREF questionnaire. The WHOQOL-BREF is a short version of the WHOQOL-100 for measurement of the impact of disease and impairment on daily activities and behavior. The WHOQOL-100 was piloted in 15 centers and field trials were conducted in 18 
countries. Zimbabwe participated in both [8], so translations in Shona for Zimbabwe are available.

The WHOQOL-BREF comprises of 26 items in four domains. These items are a subset of items derived from the World Health Organization WHOQOL-100 tool for the assessment of quality of life.

The WHOQOL-BREF consists of two parts. The first part evaluates the patient's subjective assessment of her quality of life and satisfaction with her health while the second part assesses four domains.

The first domain is on physical health. This domain looks at activities of daily living, dependence on medicinal substances and medical aids, energy and fatigue, mobility, pain and discomfort, sleep and rest and work capacity. The second domain assess psychological health and look at bodily image and appearance, negative feelings, positive feelings, self-esteem, spirituality/religion/ personal beliefs, thinking, learning, memory and concentration. The third domain assesses social relations and specifically personal relationships, social support and sexual activity. Lastly the fourth domain assesses the person's environment and looks at financial resources, freedom, physical safety and security, health and social care accessibility and quality, home environment, opportunities for acquiring new information and skills, participation in and opportunities for recreation/leisure activities, physical environment (pollution/noise/traffic/climate) and transport. Participants' responses were recorded on a Likert scale of $1-5$ on the WHOQOL-BREF tool.

The follow-up interviews were conducted mainly through telephone except for 3 participants who had home visits done between 17 June and 30 August 2020.

\subsection{Data analysis}

The quality of life data collected using the WHOQOLBREF instrument were analyzed using the associated SPSS syntax files.

The SPSS syntax file automatically checks for missing data, recodes data and computes domain scores. The four domain scores denote an individual's perception of quality of life in each particular domain. Domain scores are scaled in a positive direction (i.e. higher scores denote higher quality of life). The mean score of items within each domain was used to calculate the domain score. The mean score in each domain was obtained by computing the mean of transformed scores converted to a $0-100$ scale for each domain [8]. These were compared with the mean scores in each domain 6 months after successful fistula repair.

For the purposes of this study, a mean score of $<40$ in each domain denotes poor, 41-60 indicates moderate and $>60$ indicates good quality of life [5].
Results were presented in median plus interquartile ranges and mean \pm standard deviation (SD). The paired $t$ test was used to compare the means of scores in pretreatment and post treatment period. A $p$-value $<0.05$ was considered to be statistically significant. Significance analyses were conducted using SPSS version 16.0.

\subsection{Ethical considerations}

Permission to use the WHOQOL-BREF tool was sought and granted by WHO, Geneva. This study was approved by the national research council under license number MRCZ A2525.

All study participants gave written consent after the study processes were explained to them and their questions and concerns had been addressed. Confidentiality was maintained throughout the study.

\section{Results}

\subsection{Demographics}

A total of 26 women affected by obstetric fistula were recruited for the study. The participants' ages ranged from seventeen to 26 years with median age of 34 years (Q1: 27, Q3: 39). The median age at sexual debut was 17 years (Q1: 17, Q3:20) and 19 of the participants had their first pregnancy by age 23. Twenty-four of the participants lived in rural areas. Four participants delivered at a health facility while the other 22 did not deliver in health facilities. The majority of the participants (12 women) had been living with the fistula problem for more than 5 years, while nine had had the problem for less than a year. Three participants had HIV infection and on antiretroviral treatment, two had hypertension which was controlled on medication. All participants had transvaginal repair of fistula.

Table 1 shows the characteristics of study participants in relation to level of education, distance from health facility, type of delivery, condition of the perineum before treatment, age at first pregnancy and treatment outcomes based on physical examination. The surgical treatment success rate was $96 \%$.

Table 2 shows the scores from the two independent questions and the four domains of the WHOQOLBREF tool before and after surgical treatment.

Table 2 shows an improvement in quality of life of participants for every domain. There were significant differences in quality of life across all 26 participants $(t$ $14, d f=25: p<0.001$ ).

Table 3 shows improvements in most participants' quality of life scores for the post treatment period as compared with the pretreatment period. 
Table 1 Distribution of other demographic characteristics of participants

\begin{tabular}{|c|c|c|c|}
\hline \multicolumn{4}{|c|}{ Highest level of education } \\
\hline No education & Primary level & \multicolumn{2}{|c|}{ Secondary level and above } \\
\hline 3 & 9 & 14 & \\
\hline \multicolumn{4}{|c|}{ Distance from health facility } \\
\hline$<5 \mathrm{~km}$ & $5-10 \mathrm{~km}$ & $11-20$ km & $>20 \mathrm{~km}$ \\
\hline 3 & 8 & 7 & 8 \\
\hline \multicolumn{4}{|l|}{ Type of delivery } \\
\hline Vaginal delivery & Cesarean section & Forceps delivery & \\
\hline 23 & 2 & 1 & \\
\hline \multicolumn{4}{|c|}{ Condition of perineum before surgery } \\
\hline Intact, no sores & Mild excoriations & Serious sores & \\
\hline 4 & 5 & 17 & \\
\hline \multicolumn{4}{|c|}{ Age at first pregnancy in years } \\
\hline $15-18$ & $19-24$ & $25+$ & \\
\hline 9 & 10 & 7 & \\
\hline \multicolumn{4}{|l|}{ Baby alive } \\
\hline Yes & No & & \\
\hline 10 & 16 & & \\
\hline \multicolumn{4}{|l|}{ Treatment outcome } \\
\hline $\begin{array}{l}\text { Fistula closed and } \\
\text { dry }\end{array}$ & $\begin{array}{l}\text { Fistula closed } \\
\text { but still incon- } \\
\text { tinent }\end{array}$ & Fistula not closed & \\
\hline 22 & 2 & 2 & \\
\hline
\end{tabular}

\section{Discussion}

The success rate of treatment was noted to be high (96\%), with 24 out of 26 women having their fistula closed, although two of these, still had stress incontinence. Such high success rates have also been realized in other studies when fistula repairs are performed by experienced specialists $[5,10]$. Although 24 participants had closed fistulas, two still experienced stress incontinence. Stress incontinence has also been noted to be a problem after successful repair of fistula in other studies on experiences after fistula repair [11]. This condition is usually corrected by pelvic muscles exercises and bladder trainings $[1,12]$.
There was a significant improvement of quality of life scores in all domains of the WHOQOL-BREF assessment.

In the physical health domain, the significant improvement in quality of life scores signifies that participants rated the quality of their physical health before the treatment as being lower than after treatment. Regaining continence resulted in no more continuous wetness and sores on the vulva and thighs. The high rating of quality of physical health after treatment points to the fact that indeed women living with obstetric fistula encounter challenges in their physical health. This finding is in tandem with findings from other studies on quality of life of obstetric fistula survivors before and after treatment [5, $10,13,14]$.

In the psychological domain, a significant improvement in WHOQOL BREF score was also noted. The realization that they were now free from wetness and bad odor together with the improvement in physical health led the fistula survivors to regain self-esteem and self-confidence, necessary for good psychological health. Participants reported great improvements in sleep patterns, ability to concentrate and focus and improvement in how they feel about themselves. All these attributes are essential for good mental state.

On the social life front, the quality of life score also significantly improved from 18 before treatment to 75 after treatment. Of the 4 domains, this domain scored worst before treatment. This may explain the finding that participants experienced substantial amount of stigma, perceived stigma and actual stigma before treatment.

The participants reported improvement in interpersonal relationships as well as in sexual relationships. The cessation of urine incontinence and the healing of perineal sores may have resulted in good hygiene leading to improvement in capacity to do work, social standing and confidence. This improves social attraction and liking. Singh et al. [5] support this finding from their study in India. Social relationships with family had also improved. This finding was in contradiction with findings of a study conducted in Kenya in a study on experiences of fistula survivors where it was noted that women had not fully

Table 2 Comparison of quality of life mean scores for participants before and after treatment

\begin{tabular}{|c|c|c|c|c|}
\hline Domain & Domain description & $\begin{array}{l}\text { Mean score before treatment } \\
\text { [score (SD)] }\end{array}$ & $\begin{array}{l}\text { Mean score after treatment } \\
\text { [score (SD)] }\end{array}$ & $p$ value \\
\hline 1 & Physical & $49( \pm 1.2)$ & $80( \pm 2.0)$ & $<0.001$ \\
\hline 2 & Psychological & $26( \pm 1.2)$ & $75( \pm 2.0)$ & $<0.001$ \\
\hline 3 & Social relationships & $18( \pm 1.2)$ & $75( \pm 2.9)$ & $<0.001$ \\
\hline 4 & Environment & $34( \pm 1.0)$ & $64( \pm 1.6)$ & $<0.001$ \\
\hline Overall QOL & Overall assessment of QOL & $14( \pm 2.5)$ & $79( \pm 2.3)$ & $<0.001$ \\
\hline Health status & Satisfaction with health status & $13( \pm 2.0)$ & $77( \pm 2.5)$ & $<0.001$ \\
\hline
\end{tabular}


Table 3 Comparison of WHOQOL-BREF facet scores before and after treatment

\begin{tabular}{|c|c|c|}
\hline & $T$ statistic for differences in paired means & $p$ value \\
\hline \multicolumn{3}{|l|}{ Domain 1: Physical health } \\
\hline Activities of daily living & 5.30 & $<0.001$ \\
\hline Dependence on medicinal substances and medical aids & 1.87 & $0.073^{*}$ \\
\hline Energy and fatigue & 5.03 & $<0.001$ \\
\hline Mobility & 6.33 & $<0.001$ \\
\hline Pain and discomfort & 3.65 & 0.002 \\
\hline Sleep and rest & 7.45 & $<0.001$ \\
\hline Work capacity & 4.48 & $<0.001$ \\
\hline \multicolumn{3}{|l|}{ Domain 2: Psychological health } \\
\hline Bodily image and appearance & 8.33 & $<0.001$ \\
\hline Negative feelings & 5.17 & $<0.001$ \\
\hline Positive feelings & 12.27 & $<0.001$ \\
\hline Self esteem & 4.46 & 0.001 \\
\hline Spirituality/religion/personal beliefs & 6.32 & $<0.001$ \\
\hline Thinking, learning, memory and concentration & 9.81 & 0.004 \\
\hline \multicolumn{3}{|l|}{ Domain 3: Social relationships } \\
\hline Personal relationships & 15.26 & $<0.001$ \\
\hline Social support & 5.09 & 0.003 \\
\hline Sexual activity & 5.33 & $<0.001$ \\
\hline \multicolumn{3}{|l|}{ Domain 4: Environment } \\
\hline Financial resources & 1.36 & $0.18^{*}$ \\
\hline Freedom physical safety and security & 6.68 & $<0.001$ \\
\hline Health and social care: accessibility and quality & 2.61 & 0.015 \\
\hline Home environment & 5.31 & $<0.001$ \\
\hline Opportunities for acquiring new information and skills & 7.54 & $<0.001$ \\
\hline Participation in and opportunities for participation in recreation/leisure activities & 0.54 & $0.60^{*}$ \\
\hline Physical environment (pollution/noise/traffic/climate) & 17.32 & $<0.001$ \\
\hline Transport & 4.92 & $<0.001$ \\
\hline
\end{tabular}

* Not statistically significant, $p$ value greater than 0.05

reintegrated into society for fear of rejection by the community [15].

The environment domain included questions on how the participants assessed availability of money for their personal use, ability to participate in leisure activities and ability to perform activities of daily living among others. The WHOQOL-BREF score for this domain improved significantly. The scores for availability of money and opportunity to participate in leisure activities did not show significant changes but participants rated other aspects of the domain i.e. ability to perform activities of daily living on their own and being able to move from place to another highly.

The post treatment score for overall quality of life improved for the participants of this study. It is logical, considering that women in this study had suffered pain and other consequences of uncontrollable leakage of urine, persistent bad smell, sores and stigma associated with the presence of fistula, now with the successful repair for the majority of the participants, the score for overall quality of life went up as expected.

The same sentiments were reiterated by studies on the impact of fistula to survivors, where it was noted that the presence of fistula resulted in limitations on mobility, engagement in leisure activities and performance of daily chores [14, 16-19].

\subsection{Study limitations}

Data were not stratified by comorbidities to determine how much they contributed to the quality of life scores observed during the study.

\section{Conclusion}

With the above findings, this study concludes that obstetric fistula negatively affects the physical, psychological, social, environmental and general health of affected women. Successful surgical treatment of the 
fistula problem offers relief from the consequences of obstetric fistula in all aspects.

\subsection{Recommendations}

Considering the findings of this study, it is recommended that women affected by obstetric fistula need to be identified and treated surgically to repair the fistula so as to improve their quality of life. Fistula care programs need to be strengthened to able to provide timely treatment of fistula so as to minimize the negative effects of the condition to quality of life of survivors.

\section{Supplementary information}

The online version contains supplementary material available at https://doi. org/10.1186/s12301-020-00118-6.

Additional file 1. Obstetric fistula community surveillance tool.

\section{Abbreviations}

WHO: World Health Organization; UNFPA: United Nations Population Fund; VVF: Vesicovaginal fistula; ICS: International Continence Society; WHOQOL: World Health Organization Quality of Life assessment tool; WHOQOL-BREF: World Health Organization Quality of Life assessment tool short version.

\section{Acknowledgements}

The authors acknowledge assistance with data analysis by Mavis P. Dembedza.

\section{Authors' contributions}

CC developed the concept note and the protocol. She did data collection and analysis. She did manuscript writing. IS did protocol development and manuscript writing. SPM did protocol writing and manuscript writing. MM also did protocol development and manuscript writing. All authors read and approved the final manuscript.

\section{Funding}

This study has no funding.

\section{Availability of data and materials}

The datasets used and/or analyzed during the current study are available from the corresponding author on reasonable request.

\section{Ethical approval and consent to participate}

This study was approved by the Medical Research Council of Zimbabwe (MRCZ) under license number MRCZ A2525. Permission to use the WHOQOLBREF tool was sought and granted by WHO, Geneva. All study participants gave written informed consent after the study processes were explained to them and their questions and concerns addressed. Participants of this study were all above 16 years of age so they consented on their own. Confidentiality was maintained throughout the study.

\section{Consent for publication}

Not applicable.

\section{Competing interests}

The authors declare that they have no competing interests

\section{Author details}

${ }^{1}$ Department of Health Sciences, College of Health, Agriculture and Natural Sciences, Africa University, Mutare, Zimbabwe. ${ }^{2}$ Department of Obstetrics and Gynecology, College of Health Sciences, University of Zimbabwe, Harare, Zimbabwe.
Received: 19 November 2020 Accepted: 28 December 2020

Published online: 09 January 2021

\section{References}

1. The World Health Organization (2017) Obstetric fistula guiding principels for clinical managent and programme development. WHO Press, Geneva. https://www.afro.who.int/sites/default/files/2017-06/mps\%20 Fistula2.pdf. Accessed 1 May 2020

2. UNFPA (2015) The maternal health thematic fund 2014 annual report: improving maternal health, surging toward the 2015 deadline. https:// www.unfpa.org/sites/default/files/pub-pdf/MHTF\%20annual\%20rep ort\%20for\%20WEB_0.pdf. Accessed 1 Jun 2019

3. Polan ML, Sleemi A, Bedane MM, Lozo S, Morgan MA. Obstetric Fistula. In: Debas HT, Donkor P, Gawande A, Jamison DT, Kruk ME, Mock CN (eds) Essential surgery: disease control priorities, 3rd edn, vol 1. The International Bank for Reconstruction and Development/The World Bank, Washington (DC). http://www.ncbi.nlm.nih.gov/books/NBK33 3495/. Accessed 7 Dec 2020

4. Hebbar S, Pandey H, Chawla A (2015) Understanding King's Health Questionnaire (KHQ) in assessment of female urinary incontinence. Int J Res Med Sci 3:531-538

5. Singh V, Jhanwar A, Mehrotra S, Paul S, Sinha RJ (2015) A comparison of quality of life before and after successful repair of genitourinary fistula: Is there improvement across all the domains of WHOQOL-BREF questionnaire? Afr J Urol 21(4):230-234

6. Hebbar S, Pandey H, Chawla A (2015) Understanding King's Health Questionnaire (KHQ) in assessment of female urinary incontinence. Springer. https://www.springer.com/journal/192/submission-guide lines. Accessed 28 May 2020

7. Fultz NH, Burgio K, Diokno AC, Kinchen KS, Obenchain R, Bump RC (2003) Burden of stress urinary incontinence for community-dwelling women. Am J Obstet Gynecol 189(5):1275-1282

8. World Health Organization (2016) World Health Organization Quality of Life- BREF (WHOQOL-BREF). Spinal Cord Injury Research Evidence. http://scireproject.com/outcome-measures/outcome-measure-tool/ world-health-organization-quality-of-life-bref-whoqol-bref/. Accessed 28 May 2020

9. Lesley Fallowfield (1992) The quality of life: the missing measurement in health care. PsycCRITIQUES 1:37

10. Mwangi J, Mutiso S, Puri M, Gatebi J (2012) Quality of life in pre- and posttreatment among obstetric fistula patients at Kisii Hospital, Kenya. Clin Health Promot Res Best Pract Patients Staff Community 2(2):59-63

11. Gebresilase YT (2014) A qualitative study of the experience of obstetric fistula survivors in Addis Ababa, Ethiopia. Int J Womens Health 6:1033

12. Khisa W, Wakasiaka S, McGowan L, Campbell M, Lavender T (2017) Understanding the lived experience of women before and after fistula repair: a qualitative study in Kenya. BJOG Int J Obstet Gynaecol 124(3):503-510

13. Dennis AC, Wilson SM, Mosha MV, Masenga GG, Sikkema KJ, Terroso KE et al (2016) Experiences of social support among women presenting for obstetric fistula repair surgery in Tanzania. Int J Womens Health 8:429

14. Umoiyoho AJ, Inyang-Etoh EC, Abah GM, Abasiattai AM, Akaiso OE (2011) Quality of life following successful repair of vesicovaginal fistula in Nigeria. Afr J Urol 21:230-234

15. Khisa AM, Nyamongo IK, Omoni GM, Spitzer RF (2019) A grounded theory of regaining normalcy and reintegration of women with obstetric fistula in Kenya. Reprod Health 16(1):29

16. Animut M, Mamo A, Abebe L, Berhe MA, Asfaw S, Birhanu Z (2019) "The sun keeps rising but darkness surrounds us": a qualitative exploration of the lived experiences of women with obstetric fistula in Ethiopia. BMC Womens Health 19(1):37

17. Bashah DT, Worku AG, Mengistu MY (2018) Consequences of obstetric fistula in sub Sahara African countries, from patients' perspective: a systematic review of qualitative studies. BMC Womens Health 18(1):106

18. Luz R, Pereira I, Henriques A, Ribeirinho AL, Valentim-Lourenço A (2017) King's Health Questionnaire to assess subjective outcomes after surgical treatment for urinary incontinence: Can it be useful? Int Urogynecol J 28(1):139-145 
19. Okoye UO, Emma-Echiegu N, Tanyi PL (2014) Living with vesico-vaginal fistula: experiences of women awaiting repairs in Ebonyi State. Nigeria. Tanzan J Health Res 16(4):322-328

\section{Publisher's Note}

Springer Nature remains neutral with regard to jurisdictional claims in published maps and institutional affiliations.
Submit your manuscript to a SpringerOpen ${ }^{\circ}$ journal and benefit from:

- Convenient online submission

- Rigorous peer review

- Open access: articles freely available online

- High visibility within the field

- Retaining the copyright to your article

Submit your next manuscript at $\boldsymbol{\nabla}$ springeropen.com 